\title{
Controlling synaptotagmin activity by electrostatic screening
}

\author{
Yongsoo Park ${ }^{1}$, Javier M Hernandez ${ }^{1}$, Geert van den Bogaart ${ }^{1}$, Saheeb Ahmed ${ }^{1}$, Matthew Holt $^{1}$, Dietmar Riedel $^{2}$ \& \\ Reinhard Jahn ${ }^{1}$
}

Exocytosis of neurosecretory vesicles is mediated by the SNARE (soluble N-ethylmaleimide-sensitive factor attachment protein receptor) proteins syntaxin-1, synaptobrevin and SNAP-25, with synaptotagmin functioning as the major $\mathrm{Ca}^{2+}$ sensor for triggering membrane fusion. Here we show that bovine chromaffin granules readily fuse with large unilamellar liposomes in a SNARE-dependent manner. Fusion is enhanced by $\mathrm{Ca}^{2+}$, but only when the target liposomes contain phosphatidylinositol-4,5bisphosphate and when polyphosphate anions, such as nucleotides or pyrophosphate, are present. $\mathrm{Ca}^{2+}$-dependent enhancement is mediated by endogenous synaptotagmin-1. Polyphosphates operate by an electrostatic mechanism that reverses an inactivating cis association of synaptotagmin-1 with its own membrane without affecting trans binding. Hence, the balancing of trans- and cis-membrane interactions of synaptotagmin-1 could be a crucial element in the pathway of $\mathrm{Ca}^{2+}$-dependent exocytosis.

Neurons communicate through exocytotic release of neurotransmitters. In the resting state, neurotransmitters are stored in synaptic vesicles or secretory granules. Upon depolarization, voltage-gated calcium channels open, resulting in an influx of $\mathrm{Ca}^{2+}$ that triggers fusion of the storage-vesicle membrane with the plasma membrane, thus releasing the neurotransmitter into the extracellular space ${ }^{1}$.

Many neurons contain, in addition to synaptic vesicles, another class of secretory vesicles, which have an electron-dense core and larger diameter and are referred to as large dense-core vesicles $(\mathrm{LDCVs})^{2}$. LDCVs correspond to the secretory vesicles of neuroendocrine cells such as the chromaffin cells of the adrenal medulla. LDCVs store proteins and peptides in addition to classical neurotransmitters ${ }^{2,3}$. In neurons, LDCVs also undergo exocytosis in response to $\mathrm{Ca}^{2+}$, but their release is regulated differently from that of synaptic vesicles, requiring bursts of action potentials ${ }^{4}$. However, the release machinery, as far as it is known, utilizes the same proteins as synaptic vesicle exocytosis, including SNAREs and synaptotagmins. Owing to the ease of access to chromaffin cells with microelectrodes, exocytosis of LDCVs in these cells has served as a model for studying the mechanism of SNARE-mediated exocytosis with electrophysiological approaches ${ }^{5}$.

Vesicle docking, activation of the fusion machinery (priming), $\mathrm{Ca}^{2+}$-dependent triggering and subsequent membrane merger are carried out by evolutionarily conserved protein complexes functioning in all neurons and neuroendocrine cells. Of these, SNARE proteins are, at present, considered the catalysts of the fusion reaction ${ }^{6-9}$. SNAREs mediating synaptic exocytosis include syntaxin-1 and SNAP-25 (at the plasma membrane) and synaptobrevin (at the vesicle membrane $)^{6,10,11}$. SNAREs are small, membrane-anchored proteins that contain one or two conserved stretches of 60-70 amino acids arranged in heptad repeats, termed SNARE motifs ${ }^{6}$. Release is triggered by an influx of $\mathrm{Ca}^{2+}$ from the extracellular space in response to depolarization. Upon triggering, the SNARE motifs form a helical complex that bridges the membranes (the 'trans' complex). Complex assembly proceeds toward the membrane anchors in the N-to-C-terminal direction, thus pulling the membranes together and initiating fusion as the SNARE complex relaxes into the 'cis' configuration ${ }^{12,13}$. Triggering is mediated by synaptotagmins, transmembrane proteins of synaptic vesicles and chromaffin granules (CGs) containing two $\mathrm{Ca}^{2+}$-binding $\mathrm{C} 2$ domains-C2A and $\mathrm{C} 2 \mathrm{~B}$ - that bind three or two $\mathrm{Ca}^{2+}$ ions, respectively ${ }^{14,15}$. Synaptotagmins interact with SNAREs and acidic lipids. Both interactions are widely considered essential for their function. However, how exactly synaptotagmins accelerate fusion is unclear. We have recently shown that interactions with vesicular acidic lipids seem to inactivate synaptotagmins. Incorporation of synaptotagmin-1 into liposomes containing 20\% phosphatidylserine (PS) results in binding of synaptotagmin-1 to its own membrane (cis binding), preventing trans binding to target membranes containing acidic phospholipids and/or SNARE proteins ${ }^{16,17}$. Native vesicles contain $\sim 15 \%$ acidic phospholipids ${ }^{18}$, including PS and phosphatidylinositol (PI). It is not known how the inactivating cis association of synaptotagmins is prevented in vivo ${ }^{16,19,20}$.

Here, we have investigated the role of cis and trans binding of synaptotagmin- 1 on $\mathrm{Ca}^{2+}$-dependent fusion in vitro. To prevent problems that could arise from the differences between artificial and biological membranes, we used purified bovine CGs as one of the fusion partners. Our data show that CGs fuse with liposomes carrying syntaxin-1A and SNAP-25A in a SNARE-dependent manner. $\mathrm{Ca}^{2+}$ increased

${ }^{1}$ Department of Neurobiology, Max Planck Institute for Biophysical Chemistry, Göttingen, Germany. ${ }^{2}$ Facility for Electron Microscopy, Max Planck Institute for Biophysical Chemistry, Göttingen, Germany. Correspondence should be addressed to R.J. (rjahn@gwdg.de). 
the rate of fusion severalfold, but only when PI 4,5-bisphosphate $\left(\mathrm{PI}(4,5) \mathrm{P}_{2}\right)$ was present in the target membrane and, notably, when ATP was included in the buffer. No ATP hydrolysis was required, and the stimulatory effect of ATP was attributed to an electrostatic effect that prevents the inactivating cis binding of synaptotagmin C2 domains to the vesicle membrane, thereby allowing endogenous synaptotagmins to interact in trans with the lipids and/or SNAREs in the target membrane.

\section{RESULTS}

\section{Characterization of purified chromaffin granules}

To reconstitute CG fusion in vitro, we purified CGs from bovine adrenal medulla using a modified protocol in which centrifugation in a continuous sucrose-density gradient is the final purification step (Supplementary Fig. 1). We confirmed the purity of the CGs by western blots, which showed that the final fraction was depleted of markers of other organelles (Fig. 1a). VAMP-4, a SNARE found only on immature secretory granules ${ }^{21}$, was also removed during purification (Supplementary Fig. 1c), indicating that our protocol yields mature CGs at high purity.

Cryo-EM of purified CGs revealed a heterogeneous size distribution (Fig. 1b), with an average diameter of $167.7 \pm 14.3 \mathrm{~nm}$ (s.e.m.). Size heterogeneity of CGs in chromaffin cells has been observed previously ${ }^{22}$, with diameters of 100-500 nm, but the average diameter $(356 \mathrm{~nm})^{23}$ is larger than that found in our study. This is probably because of the inclusion, in the previous studies, of immature CGs, which are known to be larger. CGs become smaller and more condensed through the removal of water and the 'shedding' of membrane during the maturation process ${ }^{24,25}$. Our data show that our protocol yields mainly mature CGs at high purity.

\section{SNARE-dependent fusion of chromaffin granules}

We investigated whether purified CGs are able to fuse with large unilamellar liposomes (LUVs) containing SNAP-25A and syntaxin-1A. Proteoliposomes containing $N$-(7-nitro-2,1,3-benzoxadiazol-4-yl) (NBD)- and rhodamine-labeled phospholipids were reconstituted with a stabilized acceptor complex known as the $\Delta \mathrm{N}$ complex: a preformed complex of syntaxin-1A (lacking the $\mathrm{N}$-terminal Habc domain) and SNAP-25A (containing a C-terminal fragment of synaptobrevin residues 49-96 (Syb 49-96 $\left._{1}\right)$ ) (ref. 26). The lipid composition of liposomes was $45 \%$ phosphatidylcholine, $15 \%$ phosphatidylethanolamine, $10 \%$ PS, $25 \%$ cholesterol, $4 \% \mathrm{PI}$ and $1 \% \mathrm{PI}(4,5) \mathrm{P}_{2}$. Fusion was monitored by a lipid-mixing assay in which fluorescence resonance energy transfer (FRET) between the two fluorophore-labeled lipids is reduced as a result of fusion with unlabeled lipids, leading to lipid dilution and dequenching of the donor fluorophore ${ }^{27}$.

We observed robust fusion when we combined CGs with proteoliposomes containing the SNARE acceptor complex (Fig. 1c and Supplementary Fig. 2a). Membrane fusion was SNARE specific, as shown by competitive inhibition by a soluble fragment of synaptobrevin $\left(\mathrm{Syb}_{1-96}\right)$ or a soluble complex of $\mathrm{H} 3$ domain of syntaxin $1 \mathrm{~A}$ (SyxH3) and SNAP-25A and by incubation with the light chain of tetanus neurotoxin (TeNT), a protease selectively cleaving synaptobrevin (Fig. 1c and Supplementary Fig. 2a,b). Furthermore, endogenous synaptobrevin in the CG membrane was capable of forming SNARE complexes, as shown by SDS-PAGE, in which synaptobrevin assembled in the ternary SNARE complex was found to be resistant to cleavage by TeNT light chain (Fig. 1d). Lysophosphatidylcholine, which destabilizes the negative curvature of stalk-type fusion intermediates ${ }^{28}$ by inducing positive curvature, inhibited CG fusion in a dose-dependent manner (Fig. 1e and Supplementary Fig. 2c). We obtained similar results when we monitored content mixing (using a FRET-based assay) instead of lipid mixing (Supplementary Fig. 2d,e), indicating that SNARE-dependent fusion of CGs is complete and not arrested at hemifusion.

\section{$\mathrm{Ca}^{2+}$ enhances fusion in the presence of ATP}

Exocytosis of CGs is triggered, like that of synaptic vesicles, by an increase in intracellular $\mathrm{Ca}^{2+}$. We therefore investigated whether addition of $\mathrm{Ca}^{2+}$ influenced fusion between purified CGs and SNARE-containing proteoliposomes. We observed a slight but substantial inhibitory effect at $\mathrm{Ca}^{2+}$ concentrations $>300 \mu \mathrm{M}$ (Fig. 2a a

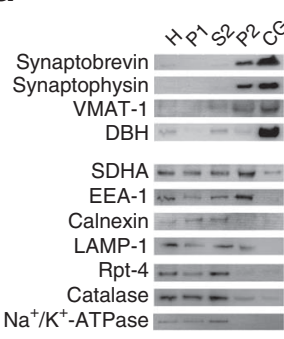

b

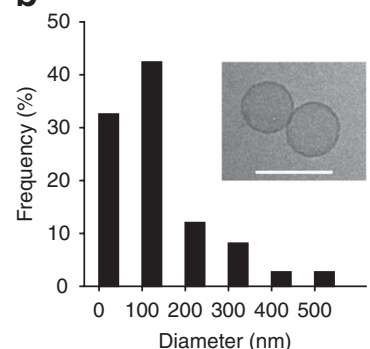

c

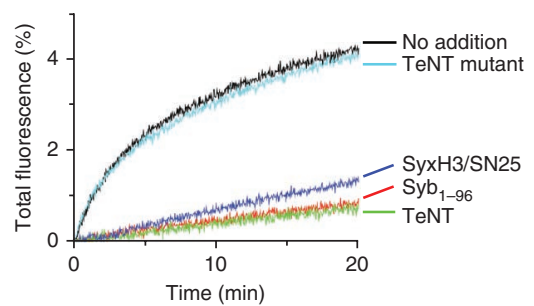

d

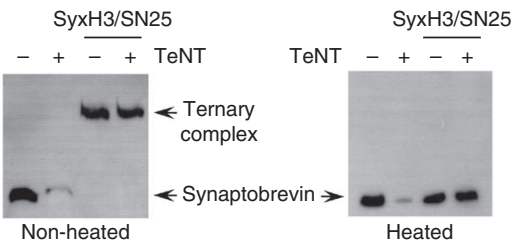

e

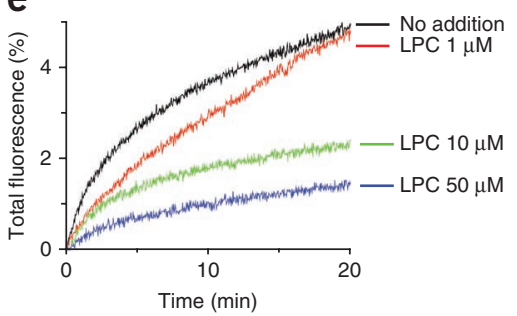

Figure 1 SNARE-dependent fusion of CGs with LUVs. (a) Western blot analysis of marker proteins specific to CGs (synaptobrevin, synaptophysin, vesicular monoamine transporter-1 (VMAT-1) and dopamine-beta-hydroxylase (DBH)); mitochondria (succinate dehydrogenase complex subunit $A$ $(\mathrm{SDH} A)$ ); endoplasmic reticulum (calnexin); early endosomes (early endosome-associated protein 1 (EEA-1)); lysosomes (lysosomal-associated membrane protein 1 (LAMP-1)); proteasomes (26S proteasome AAA-ATPase subunit RPT4 (Rpt-4)); peroxisomes (catalase) and plasma membrane $\left(\mathrm{Na}^{+} / \mathrm{K}^{+}\right.$-ATPase). See Online Methods for details; see Supplementary Figure 1 for fractionation scheme and complete blots. P1, nuclei and cell debris; S1, supernatant; P2, crude CG fraction. (b) Size distribution of CGs as determined by cryo-EM $(n=74)$. Inset, image of typical CGs; scale bar, $200 \mathrm{~nm}$. (c) Fusion of purified CGs with LUVs containing a stabilized SNARE acceptor complex as measured by fluorescence dequenching assay. Preincubation of LUVs with Syb ${ }_{1-96}$ and of CGs with SyxH3-SNAP-25A (SyxH3/SN25) completely blocked lipid mixing. Lipid mixing was also inhibited when CGs were preincubated with TeNT light chain but not when they were incubated with an inactive TeNT light-chain mutant. (d) Immunoblot detection of endogenous synaptobrevin, dissociated or in ternary SNARE complexes, after incubation of CGs with excess SyxH3-SN25 (or without (two left lanes), with (+) or without (-) TeNT, heated or non-heated. (e) Fluorescence values normalized as percentage of maximum donor fluorescence after incubation with $0.1 \%$ Triton $\mathrm{X}$ - 100 detergent. Lysophosphatidylcholine (LPC) was added before the fusion reaction; no addition, basal fusion without LPC. 
a
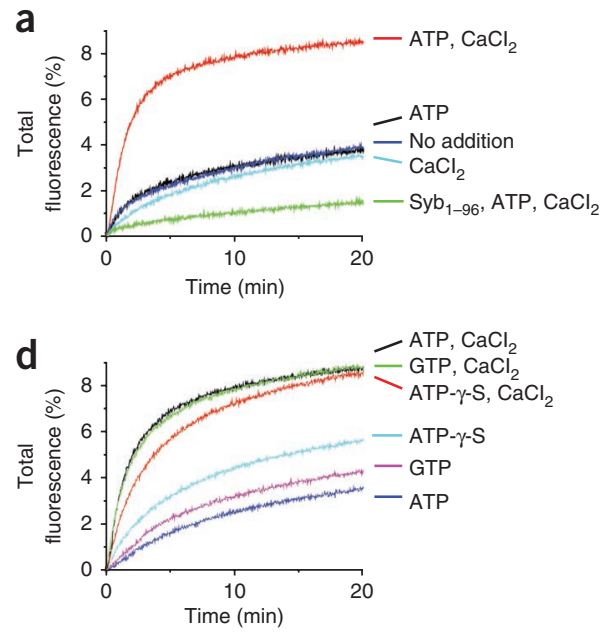

b
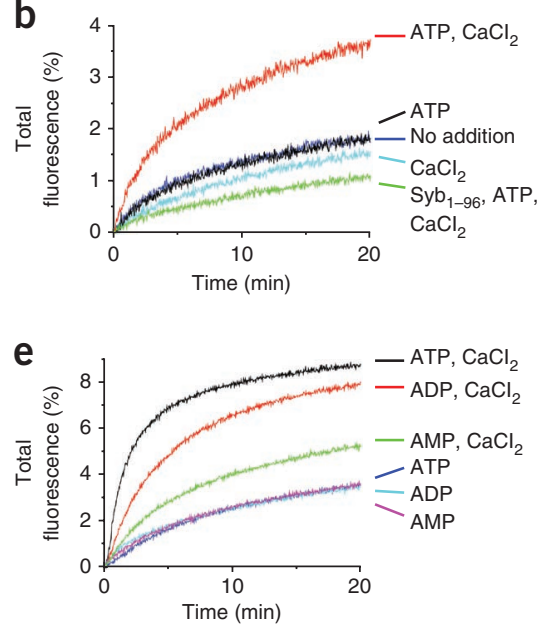
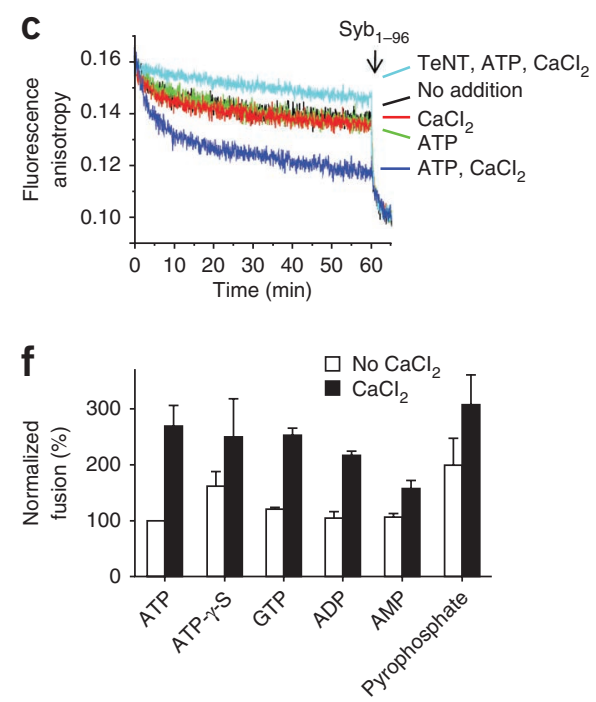

Figure 2 Role of polyphosphates in $\mathrm{Ca}^{2+}$-dependent vesicle fusion as measured by a lipid-mixing assay. (a) $\mathrm{Ca}^{2+}\left(\mathrm{CaCl}_{2}\right)$ enhances $\mathrm{CG}$ fusion with LUVs containing the stabilized acceptor complex only in the presence of ATP. Preincubation with Syb ${ }_{1-96}$ abolished Ca ${ }^{2+}$-induced CG fusion. No addition, basal fusion without ATP or $\mathrm{Ca}^{2+}$. (b) Fusion of purified synaptic vesicles from rat brain was also enhanced by Ca ${ }^{2+}$. Labels as in a. (c) TeNT cleaves synaptobrevin, and co-treatment with ATP and $\mathrm{Ca}^{2+}$ blocks SNARE assembly. At the end of each reaction, we added unlabeled Syb $1-96$ to displace all remaining Syb $49-96$. No addition, basal fusion without ATP or $\mathrm{Ca}^{2+}$. (d) ATP hydrolysis is not required for Ca ${ }^{2+}$-dependent enhancement of CG fusion. ATP was replaced with ATP- $\gamma$-S or GTP. (e) $\mathrm{Ca}^{2+}$-dependent enhancement of CG fusion was also observed in the presence of ADP and AMP, but with lower efficacy. (f) Electrostatic effect of polyphosphates on CG fusion in the presence and absence of Ca ${ }^{2+}$. Data were taken at $20-m i n$ time points and normalized as the percentage of fusion in the presence of ATP. Free $\mathrm{Ca}^{2+}(84 \mu \mathrm{M})$ was used in the presence of 5 mM ATP unless otherwise indicated. All quantitative data are mean \pm s.d. from three or more independent experiments.

and Supplementary Fig. 3a,d). While screening for metabolites that could affect fusion, we noted that, in the presence of ATP, $\mathrm{Ca}^{2+}$ did not inhibit fusion but rather enhanced it substantially (Fig. 2a and Supplementary Fig. 3d). Similarly, fusion of synaptic vesicles purified from rat brain was also increased by $\mathrm{Ca}^{2+}$ in the presence of ATP but inhibited slightly in the absence of ATP (Fig. 2b), in agreement with earlier experiments ${ }^{16,19,20,29}$. We saw no such enhancement when we used $\mathrm{MgCl}_{2}$ instead of $\mathrm{CaCl}_{2}$ (Supplementary Fig. 3b,c). Fusion was completely blocked when we added $\mathrm{Syb}_{1-96}$ as a competitive inhibitor, confirming that $\mathrm{Ca}^{2+}$-enhanced fusion is mediated by SNARE proteins. Enhancement occurred whether ATP and $\mathrm{Ca}^{2+}$ were added simultaneously or sequentially (Supplementary Fig. 4a). Furthermore, we observed the same rates of $\mathrm{Ca}^{2+}$-enhanced fusion under all conditions described when we replaced potassium glutamate in the buffer with potassium gluconate, $\mathrm{KCl}$ or $\mathrm{NaCl}$ (data not shown). We obtained similar results when we used liposomes containing a 2:1 full-length syntaxin-1A-SNAP-25A binary acceptor complex, although the rates were lower, as expected ${ }^{26}$ (Supplementary Fig. 4b).

To confirm that SNARE zippering occurs during fusion, we took advantage of the fact that $\mathrm{Syb}_{49-96}$, the C-terminal synaptobrevin fragment that stabilizes the $\Delta \mathrm{N}$ acceptor complex, is displaced by binding of full-length synaptobrevin from vesicle membranes, allowing SNARE complex assembly to be monitored by fluorescence anisotropy ${ }^{26}$. When we incubated CGs with liposomes containing the $\Delta \mathrm{N}$ complex with Alexa Fluor 488 -labeled $\mathrm{Syb}_{49-96}$, we observed a decrease of fluorescence anisotropy, indicating SNARE-complex zippering. Incubation with ATP and $\mathrm{Ca}^{2+}$ increased peptide displacement, whereas ATP or $\mathrm{Ca}^{2+}$ alone had no effect (Fig. 2c and Supplementary Fig. 3e), indicating that $\mathrm{Ca}^{2+}$-dependent enhancement of vesicle fusion is associated with an increase in number of assembled SNARE complexes.
We next investigated whether ATP hydrolysis is required for the stimulatory effect of $\mathrm{Ca}^{2+}$-enhanced fusion, which would suggest the involvement of an ATPase in the CG membrane. However, this turned out not to be the case, as we also observed synaptic vesicle fusion when we replaced ATP with the non-hydrolyzable analog ATP- $\gamma$-S (Fig. 2d-f and Supplementary Fig. 5c). Furthermore, we observed no effect on fusion when we replaced ATP with GTP. The electrostatic effect of ATP seems to depend primarily on number of negative charges, as ADP and AMP also enhanced $\mathrm{Ca}^{2+}$-dependent fusion, albeit with reduced efficacy (Fig. 2e,f). Finally, we tested pyrophosphate, which was as efficient as ADP (Fig. 2f), suggesting that the pyrophosphate moiety of the nucleotides, rather than the bases, is crucial for $\mathrm{Ca}^{2+}$-dependent vesicle fusion.

\section{$\mathrm{Ca}^{2+}$ enhancement depends on synaptotagmins and $\mathrm{PI}(4,5) \mathrm{P}_{2}$}

In chromaffin cells, members of the synaptotagmin family mediate fast exocytosis in response to $\mathrm{Ca}^{2+}$. Indeed, our results suggest that $\mathrm{Ca}^{2+}$-dependent enhancement of fusion is brought about by endogenous synaptotagmins in the CG membrane, which bind to negatively charged phospholipids by electrostatic interactions ${ }^{30}$, particularly in the presence of phosphoinositides ${ }^{31}$. We used two approaches to test this. In the first, we added a monoclonal antibody specific to the cytoplasmic domain of synaptotagmin-1 (ref. 32) to the fusion reaction, which resulted in partial inhibition of $\mathrm{Ca}^{2+}$-dependent enhancement but had no effect on basal fusion rate (Fig. 3a). A monoclonal antibody specific for the cytoplasmic domain of synaptotagmin-1 reduced $\mathrm{Ca}^{2+}$-dependent enhancement of CG fusion, compared to an antibody specific for the intravesicular domain of synaptotagmin-1 (Fig. 3a). In the second approach, we added increasing concentrations of a soluble cytoplasmic fragment of synaptotagmin-1 (the $\mathrm{C} 2 \mathrm{AB}$ domain) as a competitive inhibitor, which resulted in the progressive inhibition of $\mathrm{Ca}^{2+}$-dependent enhancement, again with no affect on basal fusion 

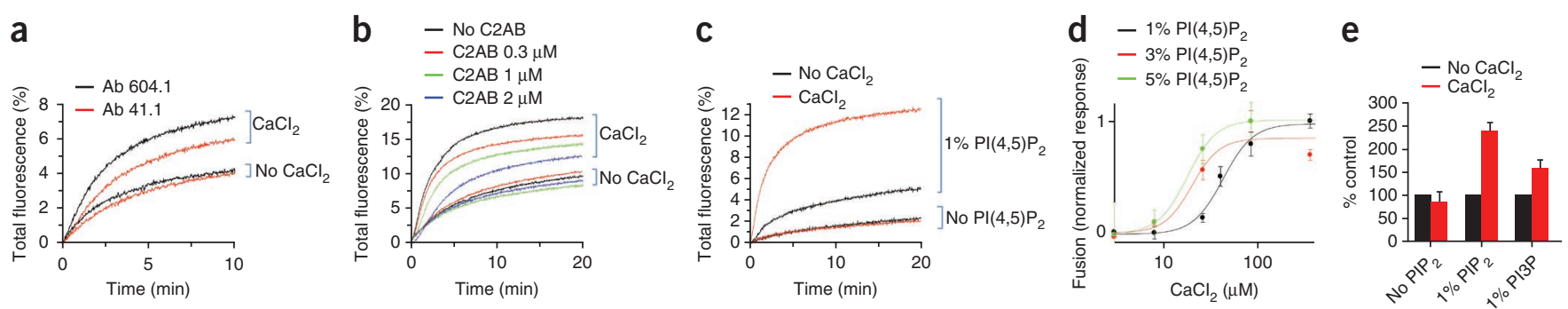

Figure 3 Roles of synaptotagmin- 1 and $\mathrm{PI}(4,5) \mathrm{P}_{2}$ in $\mathrm{Ca}^{2+}$-dependent enhancement of vesicle fusion. (a) A monoclonal antibody specific for the cytoplasmic domain of synaptotagmin-1 (Ab 41.1) reduced $\mathrm{Ca}^{2+}$-dependent enhancement of $\mathrm{CG}$ fusion, whereas no effect was observed on the basal fusion rate $\left(\right.$ no $\mathrm{CaCl}_{2}$ ). An antibody specific for the intravesicular domain of synaptotagmin-1 (Ab 604.1) was used as control. (b) Addition of a recombinant $\mathrm{C} 2 \mathrm{AB}$ domain of synaptotagmin-1 blocked $\mathrm{Ca}^{2+}$-dependent enhancement of $C G$ fusion in a dose-dependent manner. No effect on basal fusion was observed. (c) $\mathrm{PI}(4,5) \mathrm{P}_{2}$ is required for $\mathrm{Ca}^{2+}$-dependent enhancement of $\mathrm{CG}$ fusion. Omission of $\mathrm{PI}(4,5) \mathrm{P}_{2}$ from the LUV membrane reduced basal fusion and abolished $\mathrm{Ca}^{2+}$-dependent enhancement of fusion. (d) Higher $\mathrm{PI}(4,5) \mathrm{P}_{2}$ concentrations increase $\mathrm{Ca}^{2+}$ sensitivity for $\mathrm{CG}$ fusion by lowering $\mathrm{EC}_{50}$. Enhancement of vesicle fusion by

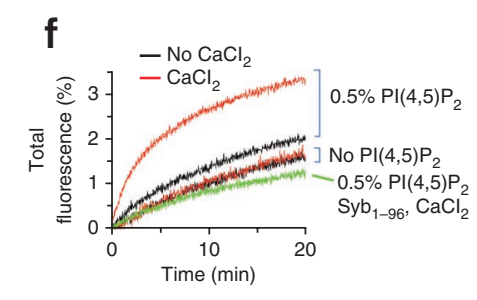
$\mathrm{Ca}^{2+}$ at $\mathrm{PI}(4,5) \mathrm{P}_{2}$ concentrations of $1 \%(40.1 \pm 2 \mu \mathrm{M}), 3 \%(20 \pm 8 \mu \mathrm{M})$ and $5 \%(17.8 \pm 8 \mu \mathrm{M})$ in LUV membranes (apparent $\mathrm{EC}_{50}$ values for Ca ${ }^{2+}$ in parentheses). $\mathrm{Ca}^{2+}$-dependent fusion was normalized to present the maximum fusion as 1 (see Online Methods for details). All quantitative data are mean \pm s.d. from three or more independent experiments. (e) $\mathrm{Ca}^{2+}$-dependent enhancement of vesicle fusion was quantified as a percentage of basal fusion in the presence of $\mathrm{PI}(4,5) \mathrm{P}_{2}\left(\mathrm{PIP}_{2}\right)$ and $\mathrm{PI3P}$. (f) Purified synaptic vesicles from rat brain also require $\mathrm{PI}(4,5) \mathrm{P}_{2}$ in the target $\mathrm{LUVs}$ for $\mathrm{Ca}^{2+}$-dependent enhancement of fusion. Free $\mathrm{Ca}^{2+}(84 \mu \mathrm{M})$ was used in the presence of $5 \mathrm{mM}$ ATP unless otherwise indicated. All quantitative data are mean \pm s.d.

rates (Fig. 3b). Together, these findings suggest that the $\mathrm{Ca}^{2+}$ effect on fusion is mediated by endogenous synaptotagmin-1, although we cannot exclude the possibility that other $\mathrm{Ca}^{2+}$ sensors (such as synaptotagmin-7) that might contribute to the effect may be present. To further verify the involvement of synaptotagmin-1, we examined whether acceleration of fusion is dependent on the presence of $\mathrm{PI}(4,5) \mathrm{P}_{2}$ in the target membrane. In chromaffin cells, plasma-membrane levels of $\mathrm{PI}(4,5) \mathrm{P}_{2}$ regulate priming ${ }^{33}$ by controlling the size of the releasable CG pool ${ }^{34}$. PI $(4,5) \mathrm{P}_{2}$ is also important for $\mathrm{Ca}^{2+}$-dependent vesicle fusion owing to its enhancement of $\mathrm{Ca}^{2+}$ affinity of synaptotagmin-1 (ref. 35), and it accumulates heavily at sites of docked vesicles ${ }^{36}$. Notably, in the absence of $\mathrm{PI}(4,5) \mathrm{P}_{2}$, the basal fusion rate was lower, and no $\mathrm{Ca}^{2+}$-dependent enhancement was observed (Fig. 3c). To gain more insight into the dependence of the $\mathrm{Ca}^{2+}$ effect on $\mathrm{PI}(4,5) \mathrm{P}_{2}$, we did titrations at different $\mathrm{PI}(4,5) \mathrm{P}_{2}$ concentrations in the target membrane (Fig. 3d). As $\mathrm{PI}(4,5) \mathrm{P}_{2}$ concentrations increased, lower $\mathrm{Ca}^{2+}$ concentrations were required to achieve the same response. These data suggest that higher local concentrations of $\mathrm{PI}(4,5) \mathrm{P}_{2}$ enhance $\mathrm{Ca}^{2+}$ sensitivity by lowering half-maximum effective concentration $\left(\mathrm{EC}_{50}\right)$, in agreement with studies carried out with purified synaptotagmin-1 (ref. 35). Next, we exchanged PI $(4,5) \mathrm{P}_{2}$ for PI 3-phosphate (PI3P), a phosphoinositide species specifically associated with endosomal and autophagosomal membranes ${ }^{37,38} \cdot \mathrm{Ca}^{2+}$-dependent enhancement still occurred but was less robust in the presence of PI3P than in the presence of $\mathrm{PI}(4,5) \mathrm{P}_{2}$ (Fig. 3e and Supplementary Fig. 4c). We also observed enhancement in the absence of $\mathrm{PI}(4,5) \mathrm{P}_{2}$ when we added the acidic phospholipid PS, which has a net charge of -1 , at a concentration of $40 \%$ (Supplementary Fig. 4d). We obtained similar results for the dependence on $\mathrm{PI}(4,5) \mathrm{P}_{2}$ when we used synaptic vesicles instead of CGs (Fig. 3f and Supplementary Fig. 5a).

ATP prevents cis binding of synaptotagmins by charge shielding What is the mechanism by which polyphosphate anions enhance $\mathrm{Ca}^{2+}$ - and synaptotagmin-mediated fusion of CGs and synaptic a

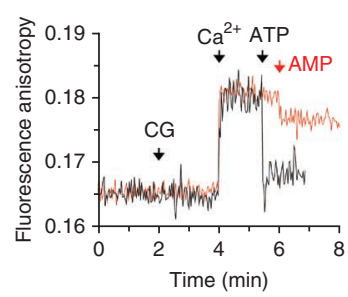

b

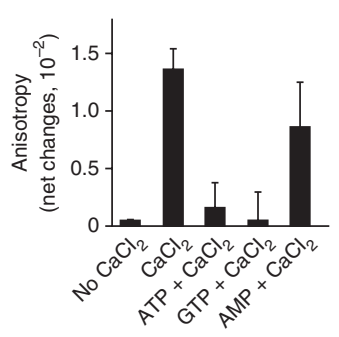

C

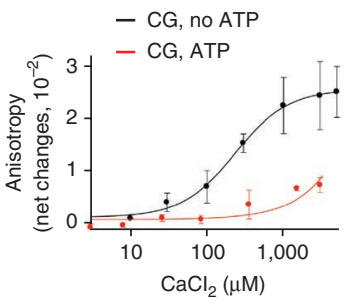

d

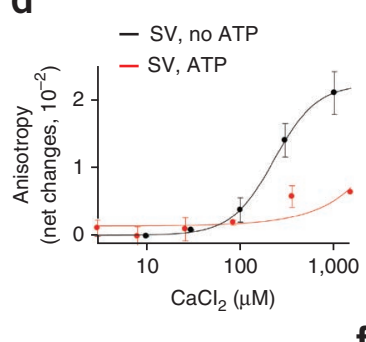

e

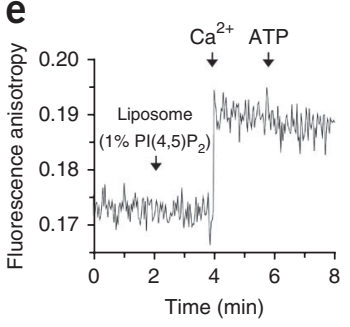

Figure 4 Effect of ATP on association of synaptotagmin-1 to the vesicle membrane. (a,b) C2AB binding to CG membranes was monitored by fluorescence anisotropy. (a) CGs were incubated with a purified fragment of synaptotagmin-1 encompassing both C2 domains (Syt ${ }_{97-421}$, Alexa Fluor 488-labeled at S342C). Binding resulted in an increase of anisotropy due to a reduced mobility of the fluorophores ${ }^{35}$. Addition of ATP ( 5 mM) reduces free $\mathrm{Ca}^{2+}$ concentration to $84 \mu \mathrm{M}$ due to chelation; see Online Methods for details. Net changes of fluorescence anisotropy intensity by $\mathrm{Ca}^{2+}$ are shown in $\mathbf{b}$. (c,d) $\mathrm{Ca}^{2+}$-dependence of $\mathrm{C} 2 \mathrm{AB}$ binding to purified CGs (c) and synaptic vesicles (SVs; d) in the presence and absence of $5 \mathrm{mM}$ ATP. (e,f) Fluorescence anisotropy over time (e) and net changes under increasing $\mathrm{Ca}^{2+}$ concentrations (f) of membrane binding using liposomes (Lip)

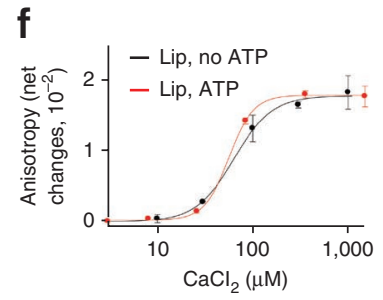
containing $1 \% \mathrm{PI}(4,5) \mathrm{P}_{2}$. All quantitative data are mean \pm s.d. from three or more independent experiments. All $\mathrm{Ca}^{2+}$-concentrations were corrected to account for ATP-dependent chelation. 
Figure 5 Effect of acidic phospholipid concentration in the liposome membrane on $\mathrm{Ca}^{2+}$-dependent binding of synaptotagmin-1. (a-c) $\mathrm{Ca}^{2+}$-dependent binding of the $\mathrm{C} 2 \mathrm{AB}$ domain was monitored by fluorescence anisotropy as in Figure 4, using LUVs containing $20 \%$ (a), $15 \%$ (b) and $10 \%$ (c) phosphatidylserine (PS). Left panels show exemplary fluorescence anisotropy traces; middle panels show net changes of anisotropy in dependence on free $\mathrm{Ca}^{2+}$ concentration; right panels show changes in dependence on $\mathrm{Ca}^{2+}$ and ATP of membrane fusion. Donor liposomes were reconstituted with full-length synaptotagmin-1 (Syt-1) and synaptobrevin-2 (Syb), whereas acceptor LUVs contained a stabilized SNARE acceptor complex (Syx/SN) and $1 \% \mathrm{PI}(4,5) \mathrm{P}_{2}$ (see diagram above panels). Data were normalized to the fusion activity measured in the absence of $\mathrm{Ca}^{2+}$ in the same experiment. All quantitative data are mean \pm s.d. from three or more independent experiments.

vesicles? Considering the data above, we hypothesized that polyphosphates such as ATP and related compounds might activate endogenous synaptotagmins in the membranes of CGs and synaptic vesicles. Previous studies have suggested that the $\mathrm{C} 2$ domains of membrane-anchored synaptotagmins might interact with their anchor membranes (cis binding), which could interfere with or even prevent binding to the target membrane ${ }^{16,17,29}$. Trans binding of synaptotagmins, however, is believed to be required for synaptotagmins to drive $\mathrm{Ca}^{2+}$-dependent exocytosis. Because we were unable to directly measure association of endogenous synaptotagmins, we used fluorescence anisotropy to monitor binding of an exogenously added $\mathrm{C} 2 \mathrm{AB}$ fragment of synaptotagmin-1, labeled with Alexa Fluor 488, to CG and synaptic vesicle membranes (Fig. 4). Addition of $\mathrm{Ca}^{2+}$ increased fluorescence anisotropy, indicating that the $\mathrm{C} 2 \mathrm{AB}$ domains of synaptotagmin- 1 bind to $\mathrm{CG}$ membranes in a $\mathrm{Ca}^{2+}$-dependent manner (Fig. $\mathbf{4 a - c}$ ). Addition of ATP or GTP after $\mathrm{Ca}^{2+}$ decreased the anisotropy signal, suggesting dissociation of the $\mathrm{C} 2 \mathrm{AB}$ fragment from the membrane. Addition of AMP had a smaller effect on anisotropy, thus mirroring the effects of AMP on fusion enhancement. We obtained very similar results when using purified synaptic vesicles instead of CGs (Fig. 4d). Increase of the free $\mathrm{Ca}^{2+}$ concentration to $>1 \mathrm{mM}$ did not affect the ability of ATP to prevent binding (Fig. 4c,d; see Online Methods for details).

Taken together, these results suggest that synaptotagmins bind to their own membranes (cis binding) in response to $\mathrm{Ca}^{2+}$, which abolishes trans interactions, in agreement with our previous observations using artificial vesicles ${ }^{17}$. To analyze in more detail the effect of ATP on the $\mathrm{Ca}^{2+}$-dependent binding of synaptotagmins to acidic membranes, we carried out binding experiments using $\mathrm{PI}(4,5) \mathrm{P}_{2}$-containing liposomes with the same composition as those used in the fusion assays. Addition of $\mathrm{Ca}^{2+}$ resulted in increased binding of the $\mathrm{C} 2 \mathrm{AB}$ domain, and this binding was not reversed by ATP (Fig. 4e,f). Cis binding of synaptotagmins is prevented by polyphosphate anions at physiological concentrations, whereas trans interactions with $\mathrm{PI}(4,5) \mathrm{P}_{2}$-containing target membranes do not seem to be inhibited.

$\mathrm{Ca}^{2+}$-dependent binding of the $\mathrm{C} 2$ domains to membranes is strongly enhanced by $\mathrm{PI}(4,5) \mathrm{P}_{2}$, but it also depends on the concentration of acidic membrane lipids such as PS or PI. We therefore
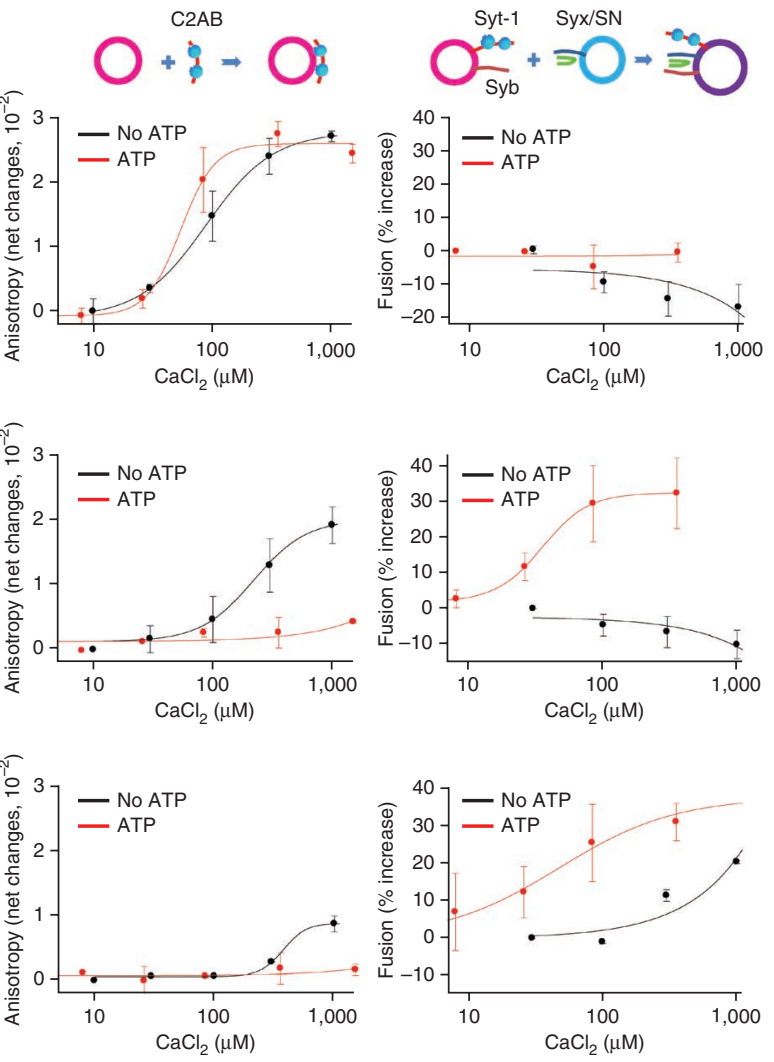

speculated that the inhibitory effect of ATP and its paralogs might be effective only at moderate concentrations of such acidic lipids. To test this, we measured $\mathrm{Ca}^{2+}$-dependent binding of synaptotagmin-1 to liposomes at increasing concentrations of PS, in the presence and absence of ATP (Fig. 5). As described previously ${ }^{31}$, the calcium sensitivity of $\mathrm{Ca}^{2+}$-dependent binding was increased with increasing PS concentrations. Notably, ATP reversed binding at PS concentrations of $10 \%$ and $15 \%$ but not $20 \%$ (for reference, synaptic vesicle membranes are $\sim 15 \%$ acidic phospholipids $)^{18}$. Indeed, the $\mathrm{Ca}^{2+}$ dose-response curves for $\mathrm{C} 2 \mathrm{AB}$ binding to CGs (Fig. 4c) and synaptic vesicles (Fig. 4d) resemble that of liposomes containing 15\% PS (Fig. 5b), indicating that a concentration of $15 \%$ PS mimics the electrostatic environment of native vesicle membranes.

Finally, we tested whether we could reproduce cis inactivation, its prevention by ATP and trans acceleration of fusion using liposomes reconstituted with synaptobrevin and synaptotagmin-1 as donor vesicles instead of purified CGs and synaptic vesicles. Specifically, we asked whether synaptotagmin-1 is capable of enhancing SNARE-mediated fusion in a $\mathrm{Ca}^{2+}$-dependent manner, and we found that it was (Fig. 5). $\mathrm{Ca}^{2+}$-dependent acceleration of fusion correlated with the prevention of cis binding by ATP (Fig. 5b, right), but we did not observe the enhancement when the membranes of the vesicles incorporating synaptotagmin-1 and synaptobrevin contained more than 20\% PS (Fig. 5a, right). These observations agree with a recent report showing that $\mathrm{Ca}^{2+}$-dependent enhancement of fusion depends on the amount of PS in synaptotagmin-1-containing liposomes ${ }^{39}$. Notably, $\mathrm{Ca}^{2+}$-dependent fusion of liposomes was less efficient than fusion of native vesicles.

\section{DISCUSSION}

Exocytosis of neurosecretory vesicles is mediated by SNAREs and triggered by $\mathrm{Ca}^{2+}$-bound synaptotagmins. However, the mechanism by which synaptotagmins enhance fusion is still unclear. In vitro 
a

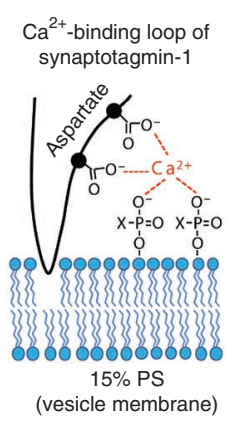

b

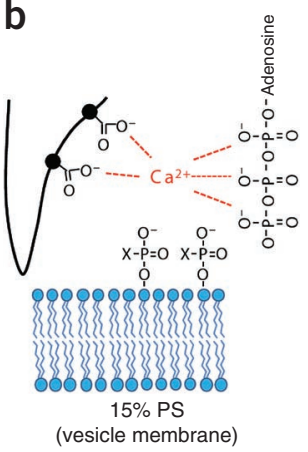

C

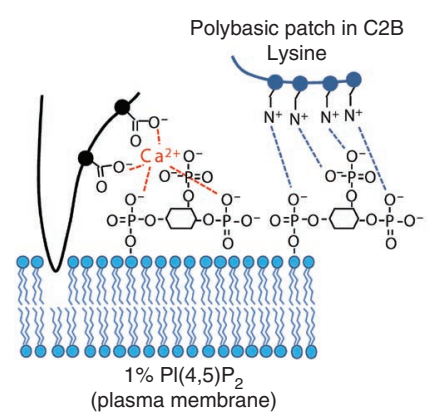

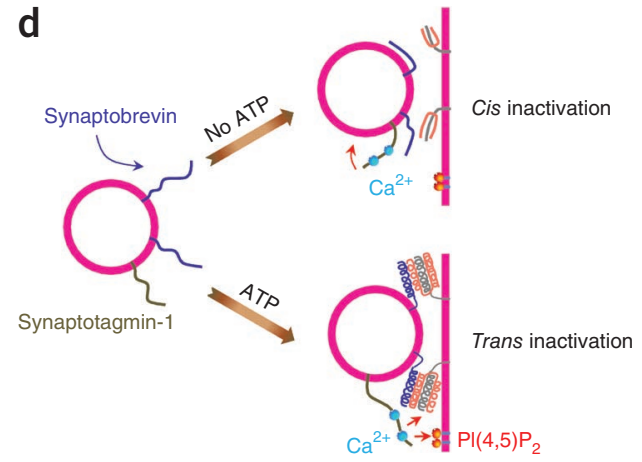

Figure 6 Effect of polyphosphates on $\mathrm{Ca}^{2+}$-dependent binding of synaptotagmins to membranes containing acidic phospholipids. (a) In the absence of ATP, synaptotagmin-1 binds to the vesicle membrane in a $\mathrm{Ca}^{2+}$-dependent manner $\left(\mathrm{EC}_{50}=233 \mu \mathrm{M} \mathrm{Ca}{ }^{2+}\right)$. Aspartate residues of the $\mathrm{C} 2$ domains (black balls) provide partial coordination for $\mathrm{Ca}^{2+}$ ions, with a more complete coordination sphere contributed by acidic membrane lipids. (b) ATP, which chelates $\mathrm{Ca}^{2+}$ with $K_{\mathrm{d}}=230 \mu \mathrm{M}$ (ref. 42), competes with $\mathrm{Ca}^{2+}$-dependent membrane binding of synaptotagmin-1 by shielding the coordination site of acidic phospholipids. (c) In the presence of $\mathrm{PI}(4,5) \mathrm{P}_{2}, \mathrm{Ca}^{2+}$ binding between the membrane and the $\mathrm{C} 2$ domains is enhanced, with an $\mathrm{EC}_{50}=56 \mu \mathrm{M}$ $\mathrm{Ca}^{2+}$ (Fig. 4f), probably because of a polybasic patch within the $\mathrm{C} 2 \mathrm{~B}$ domain that binds $\mathrm{PI}(4,5) \mathrm{P}_{2}$ on its own. The high $\mathrm{Ca}^{2+}$ affinity of $\mathrm{C} 2 \mathrm{AB}$ binding to $\mathrm{PI}(4,5) \mathrm{P}_{2}$-containing target membranes cannot be competed for by ATP. (d) In the absence of ATP, Ca $2+$ inactivates synaptotagmin- 1 through cis association (binding to vesicle membrane). ATP selectively abolishes cis binding of synaptotagmin-1, leaving the C2AB domains active to interact with the plasma membrane containing $\mathrm{PI}(4,5) \mathrm{P}_{2}$, as required for $\mathrm{Ca}^{2+}$ triggering of exocytosis.

reconstitution of SNARE-dependent membrane fusion in the presence of synaptotagmins has yielded conflicting results, with the effects of $\mathrm{Ca}^{2+}$ ranging from slight inhibition to enhancement under widely varying conditions ${ }^{16,29,39-41}$. We have previously shown that membraneanchored synaptotagmin-1 binds to its own membrane (cis binding) when acidic phospholipids are present, preventing trans interactions with the target membrane ${ }^{16,17}$. Here we have shown that cis binding of synaptotagmin-1 occurs in native CGs and synaptic vesicles, but that such cis binding is prevented by polyphosphate anions, including ATP, at physiological concentrations. ATP and analogous compounds probably operate by charge screening-that is, by competing directly with acidic membrane lipids to chelate $\mathrm{Ca}^{2+}$ and disrupt cis binding of synaptotagmins (Fig. 6). A native vesicle membrane that contains $\sim 15 \%$ acidic phospholipids has a $\mathrm{Ca}^{2+}$ affinity, with $\mathrm{EC}_{50}=233 \pm$ $29 \mu \mathrm{M}$, for binding of the C2AB domain (Fig. 4c), and ATP on its own chelates $\mathrm{Ca}^{2+}$ with a similar affinity $\left(\mathrm{EC}_{50}=230 \mu \mathrm{M}\right.$, data not shown; see also http://maxchelator.stanford.edu and ref. 42), thereby inhibiting $\mathrm{C} 2 \mathrm{AB}$ binding to the vesicle membrane. Notably, screening is effective only when the concentration of acidic phospholipids does not exceed $15 \%$ and when no $\mathrm{PI}(4,5) \mathrm{P}_{2}$ is present in the membrane. $\mathrm{PI}(4,5) \mathrm{P}_{2}$-containing target membranes have a much higher $\mathrm{Ca}^{2+}$ affinity than vesicle membranes for $\mathrm{C} 2 \mathrm{AB}$ binding $\left(\mathrm{EC}_{50}=56 \pm\right.$ $9 \mu \mathrm{M}$ ), and synaptotagmins can then act in 'trans', resulting in a major $\mathrm{Ca}^{2+}$-dependent enhancement of SNARE-dependent fusion.

Our data shed light on the mechanisms by which synaptotagmin $\mathrm{Ca}^{2+}$ sensors might operate between the vesicle and the plasma membrane. It is well established that both $\mathrm{C} 2$ domains show highly cooperative $\mathrm{Ca}^{2+}$-dependent binding to membranes containing acidic phospholipids ${ }^{15,30}$, with higher concentrations of acidic phospholipids in the membrane resulting in higher affinities ${ }^{31}$. Furthermore, synaptotagmins contain a basic patch in the $\mathrm{C} 2 \mathrm{~B}$ domain that binds to $\mathrm{PI}(4,5) \mathrm{P}_{2}$ in an at least partially $\mathrm{Ca}^{2+}$-independent manner ${ }^{43,44}$ and enhances the $\mathrm{Ca}^{2+}$ sensitivity of exocytosis ${ }^{45}$. Our data suggest that there may be a delicate balance between cis and trans binding of synaptotagmins: whereas the target membrane containing high concentrations of $\mathrm{PI}(4,5) \mathrm{P}_{2}$ allows for strong $\mathrm{Ca}^{2+}$-dependent (and partially $\mathrm{Ca}^{2+}$-independent) binding of synaptotagmins, the concentration of acidic phospholipids in the vesicle membrane seems to be adjusted to regulate cis binding of synaptotagmins.
We do not yet know whether polyphosphate-dependent screening of inactivating cis binding has a role in the regulation of $\mathrm{Ca}^{2+}$-dependent exocytosis under physiological conditions. In permeabilized neuroendocrine cells, triggering of exocytosis by $\mathrm{Ca}^{2+}$ depends on the presence of $\mathrm{ATP}^{46}$. Moreover, we have shown previously that in a cell-free preparation composed of inverted lawns of plasma membrane containing docked secretory vesicles, addition of $\mathrm{Ca}^{2+}$ elicits exocytosis, but only when ATP is present ${ }^{47}$. ATP dependence might simply reflect the involvement of ATP-using enzymes such as $\mathrm{N}$-ethylmaleimide-sensitive fusion protein or PI 4-kinase. However, it is conceivable that the strict dependence on the presence of ATP even during the $\mathrm{Ca}^{2+}$-triggering phase could also be attributable to the prevention of inactivating cis binding of synaptotagmins to the vesicle membrane.

How do these data contribute to the understanding of the stilldebated mechanism by which $\mathrm{Ca}^{2+}$-binding to synaptotagmins accelerates the rate of exocytosis by more than five orders of magnitude? Many models have been suggested for the action of synaptotagmins in the fusion pathway, such as the unblocking or activation of arrested trans SNARE complexes, generation of localized membrane curvature in the plasma membrane or perturbation of the hydrophilichydrophobic boundary of the membranes at the contact site. Although we cannot yet exclude any of these mechanisms, it has recently been suggested that synaptotagmins may act as distance regulators that pull the vesicle and the plasma membrane a bit closer upon $\mathrm{Ca}^{2+}$ triggering, thus triggering SNARE assembly and fusion ${ }^{48}$. It is possible that synaptotagmins first bind in trans to the target membrane and that this trans binding is then followed by cis binding-involving the $\mathrm{C} 2$ domains - to the vesicle membrane, shortening the distance between the membranes. Such a two-step mechanism could allow for regulation of cis binding, for example, by fine-tuning the concentration of acidic phospholipids and/or phosphorylated variants of PI in the vesicle membrane at the contact site.

\section{METHODS}

Methods and any associated references are available in the online version of the paper.

Note: Supplementary information is available in the online version of the paper. 
ACKNOWLEDGMENTS

We are indebted to G. Mieskes (Department of Neurobiology, Max Planck Institute for Biophysical Chemistry) for the arrangement of adrenal glands and logistical assistance. This work was supported by grants from the Alexander von Humboldt Foundation (to Y.P.) and the US National Institutes of Health (2 P01 GM07269406A1 to R.J.).

\section{AUTHOR CONTRIBUTIONS}

J.M.H. assisted in the generation of SNARE-containing large unilamellar liposomes and performed the light-scattering experiments. G.v.d.B. provided labeled proteins and assisted in the fluorescence anisotropy experiments. S.A. and M.H. provided purified synaptic vesicles. D.R. performed EM. Y.P. and R.J. designed the study and wrote the paper. Experiments were conducted mainly by Y.P. All authors discussed the results and commented on the manuscript.

\section{COMPETING FINANCIAL INTERESTS}

The authors declare no competing financial interests.

Published online at http://www.nature.com/doifinder/10.1038/nsmb.2375.

Reprints and permissions information is available online at http://www.nature.com/ reprints/index.html.

1. Augustine, G.J. How does calcium trigger neurotransmitter release? Curr. Opin. Neurobiol. 11, 320-326 (2001).

2. De Camilli, P. \& Jahn, R. Pathways to regulated exocytosis in neurons. Annu. Rev. Physiol. 52, 625-645 (1990).

3. Park, Y. \& Kim, K.T. Short-term plasticity of small synaptic vesicle (SSV) and large large dense-core vesicle (LDCV) exocytosis. Cell. Signal. 21, 1465-1470 (2009).

4. Neher, E. Vesicle pools and $\mathrm{Ca}^{2+}$ microdomains: new tools for understanding their roles in neurotransmitter release. Neuron 20, 389-399 (1998).

5. Neher, E. A comparison between exocytic control mechanisms in adrenal chromaffin cells and a glutamatergic synapse. Pflugers Arch. 453, 261-268 (2006).

6. Jahn, R. \& Scheller, R.H. SNAREs-engines for membrane fusion. Nat. Rev. Mol. Cell Biol. 7, 631-643 (2006).

7. Martens, S. \& McMahon, H.T. Mechanisms of membrane fusion: disparate players and common principles. Nat. Rev. Mol. Cell Biol. 9, 543-556 (2008).

8. Martin, T.F. The molecular machinery for fast and slow neurosecretion. Curr. Opin. Neurobiol. 4, 626-632 (1994).

9. Rizo, J. \& Rosenmund, C. Synaptic vesicle fusion. Nat. Struct. Mol. Biol. 15, 665-674 (2008).

10. Malsam, J., Kreye, S. \& Sollner, T.H. Membrane fusion: SNAREs and regulation. Cell. Mol. Life Sci. 65, 2814-2832 (2008).

11. Südhof, T.C. \& Rothman, J.E. Membrane fusion: grappling with SNARE and SM proteins. Science 323, 474-477 (2009).

12. Stein, A., Weber, G., Wahl, M.C. \& Jahn, R. Helical extension of the neuronal SNARE complex into the membrane. Nature 460, 525-528 (2009).

13. Wiederhold, K. \& Fasshauer, D. Is assembly of the SNARE complex enough to fuel membrane fusion? J. Biol. Chem. 284, 13143-13152 (2009).

14. Fernandez, I. et al. Three-dimensional structure of the synaptotagmin $1 \mathrm{C} 2 \mathrm{~B}$ domain: synaptotagmin-1 as a phospholipid binding machine. Neuron 32 , 1057-1069 (2001).

15. Chapman, E.R. How does synaptotagmin trigger neurotransmitter release? Annu. Rev. Biochem. 77, 615-641 (2008).

16. Stein, A., Radhakrishnan, A., Riedel, D., Fasshauer, D. \& Jahn, R. Synaptotagmin activates membrane fusion through a $\mathrm{Ca}^{2+}$-dependent trans interaction with phospholipids. Nat. Struct. Mol. Biol. 14, 904-911 (2007).

17. Vennekate, W. et al. Cis- and trans-membrane interactions of synaptotagmin-1. Proc. Natl. Acad. Sci. USA 109, 11037-11042 (2012).

18. Takamori, S. et al. Molecular anatomy of a trafficking organelle. Cell 127, 831-846 (2006).

19. Holt, M., Riedel, D., Stein, A., Schuette, C. \& Jahn, R. Synaptic vesicles are constitutively active fusion machines that function independently of $\mathrm{Ca}^{2+}$. Curr. Biol. 18, 715-722 (2008).

20. Mahal, L.K., Sequeira, S.M., Gureasko, J.M. \& Sollner, T.H. Calcium-independent stimulation of membrane fusion and SNAREpin formation by synaptotagmin 1 . J. Cell Biol. 158, 273-282 (2002).
21. Eaton, B.A., Haugwitz, M., Lau, D. \& Moore, H.P. Biogenesis of regulated exocytotic carriers in neuroendocrine cells. J. Neurosci. 20, 7334-7344 (2000).

22. Grabner, C.P., Price, S.D., Lysakowski, A. \& Fox, A.P. Mouse chromaffin cells have two populations of dense-core vesicles. J. Neurophysiol. 94, 2093-2104 (2005).

23. Plattner, H., Artalejo, A.R. \& Neher, E. Ultrastructural organization of bovine chromaffin cell cortex-analysis by cryofixation and morphometry of aspects pertinent to exocytosis. J. Cell Biol. 139, 1709-1717 (1997).

24. Kim, T., Gondre-Lewis, M.C., Arnaoutova, I. \& Loh, Y.P. Dense-core secretory granule biogenesis. Physiology (Bethesda) 21, 124-133 (2006).

25. Meldolesi, J., Chieregatti, E. \& Luisa Malosio, M. Requirements for the identification of dense-core granules. Trends Cell Biol. 14, 13-19 (2004).

26. Pobbati, A.V., Stein, A. \& Fasshauer, D. N- to C-terminal SNARE complex assembly promotes rapid membrane fusion. Science 313, 673-676 (2006).

27. Struck, D.K., Hoekstra, D. \& Pagano, R.E. Use of resonance energy transfer to monitor membrane fusion. Biochemistry 20, 4093-4099 (1981).

28. Chernomordik, L.V. et al. Lysolipids reversibly inhibit $\mathrm{Ca}^{2+}$, GTP- and $\mathrm{pH}$-dependent fusion of biological membranes. FEBS Lett. 318, 71-76 (1993).

29. Lee, H.K. et al. Dynamic $\mathrm{Ca}^{2+}$-dependent stimulation of vesicle fusion by membraneanchored synaptotagmin-1. Science 328, 760-763 (2010).

30. Davletov, B.A. \& Sudhof, T.C. A single C2 domain from synaptotagmin-1 is sufficient for high-affinity $\mathrm{Ca}^{2+}$-phospholipid binding. J. Biol. Chem. 268, 26386-26390 (1993).

31. Zhang, X., Rizo, J. \& Sudhof, T.C. Mechanism of phospholipid binding by the C2Adomain of synaptotagmin-1. Biochemistry 37, 12395-12403 (1998).

32. Chapman, E.R. \& Jahn, R. Calcium-dependent interaction of the cytoplasmic region of synaptotagmin with membranes. Autonomous function of a single C2-homologous domain. J. Biol. Chem. 269, 5735-5741 (1994).

33. Di Paolo, G. \& De Camilli, P. Phosphoinositides in cell regulation and membrane dynamics. Nature 443, 651-657 (2006)

34. Milosevic, I. et al. Plasmalemmal phosphatidylinositol-4,5-bisphosphate level regulates the releasable vesicle pool size in chromaffin cells. J. Neurosci. 25 2557-2565 (2005).

35. Radhakrishnan, A., Stein, A., Jahn, R. \& Fasshauer, D. The $\mathrm{Ca}^{2+}$ affinity of synaptotagmin-1 is markedly increased by a specific interaction of its $C_{2} B$ domain with phosphatidylinositol-4,5-bisphosphate. J. Biol. Chem. 284, 25749-25760 (2009).

36. van den Bogaart, G. et al. Membrane-protein sequestering by ionic protein-lipid interactions. Nature 479, 552-555 (2011).

37. Botelho, R.J., Scott, C.C. \& Grinstein, S. Phosphoinositide involvement in phagocytosis and phagosome maturation. Curr. Top. Microbiol. Immunol. 282, 1-30 (2004).

38. Gillooly, D.J. et al. Localization of phosphatidylinositol-3-phosphate in yeast and mammalian cells. EMBO J. 19, 4577-4588 (2000).

39. Lai, Y. \& Shin, Y.K. The importance of an asymmetric distribution of acidic lipids for synaptotagmin-1 function as a $\mathrm{Ca}^{2+}$ sensor. Biochem. J. 443, 223-229 (2012).

40. Bhalla, A., Chicka, M.C., Tucker, W.C. \& Chapman, E.R. Ca ${ }^{2+}$-synaptotagmin directly regulates t-SNARE function during reconstituted membrane fusion. Nat. Struct. Mol. Biol. 13, 323-330 (2006).

41. Kyoung, M. et al. In vitro system capable of differentiating fast $\mathrm{Ca}^{2+}$-triggered content mixing from lipid exchange for mechanistic studies of neurotransmitter release. Proc. Natl. Acad. Sci. USA 108, E304-E313 (2011).

42. Wilson, J.E. \& Chin, A. Chelation of divalent cations by ATP, studied by titration calorimetry. Anal. Biochem. 193, 16-19 (1991).

43. Kuo, W., Herrick, D.Z., Ellena, J.F. \& Cafiso, D.S. The calcium-dependent and calcium-independent membrane binding of synaptotagmin-1: two modes of $\mathrm{C} 2 \mathrm{~B}$ binding. J. Mol. Biol. 387, 284-294 (2009).

44. Vrljic, M. et al. Post-translational modifications and lipid-binding profile of insect cell-expressed full-length mammalian synaptotagmin-1. Biochemistry $\mathbf{5 0}$ 9998-10012 (2011)

45. Li, L. et al. Phosphatidylinositol phosphates as co-activators of $\mathrm{Ca}^{2+}$ binding to $\mathrm{C} 2$ domains of synaptotagmin-1. J. Biol. Chem. 281, 15845-15852 (2006).

46. Baker, P.F. \& Knight, D.E. Calcium-dependent exocytosis in bovine adrenal medullary cells with leaky plasma membranes. Nature 276, 620-622 (1978).

47. Barszczewski, M. et al. A novel site of action for $\alpha$-SNAP in the SNARE conformational cycle controlling membrane fusion. Mol. Biol. Cell 19, 776-784 (2008).

48. van den Bogaart, G. et al. Synaptotagmin-1 may be a distance regulator acting upstream of SNARE nucleation. Nat. Struct. Mol. Biol. 18, 805-812 (2011). 


\section{ONLINE METHODS}

Materials. Calcein, ATP, ADP, AMP, GTP, ATP- $\gamma$-S and pyrophosphate were purchased from Sigma (St. Louis, MO). L- $\alpha$-Lysophosphatidylcholine (LPC) and other lipids were from Avanti (Alabaster, AL). Antibodies to synaptobrevin (clone number 69.1), synaptophysin (clone number 7.2), synaptotagmin-1 (monoclonal antibodies 41.1 and 604.1) and VAMP-4 (catalog no. 136 002) were from Synaptic Systems (Göttingen, Germany). Antibodies to SDHA (catalog no. ab14715), EEA-1 (catalog no. ab2900), calnexin (catalog no. ab10286), LAMP-1 (catalog no. ab24170), Rpt-4 (catalog no. ab22639), catalase (catalog no. ab1877) and $\mathrm{Na}^{+} / \mathrm{K}^{+}$-ATPase (catalog no. ab7671) were from Abcam (Cambridge, MA). All antibodies were diluted to 1:2,000 for use.

Purification of chromaffin granules and synaptic vesicles. CGs were purified as previously described ${ }^{49}$, with several modifications. Fresh bovine adrenal glands were obtained from a local slaughterhouse. After the cortex and fat were trimmed away, the medullae were minced with a scissor in $300-\mathrm{mM}$ sucrose buffer $(300 \mathrm{mM}$ sucrose, $20 \mathrm{mM}$ HEPES ( $\mathrm{pH}$ 7.4) adjusted with $\mathrm{KOH}$ ) and then homogenized using a cooled glass-Teflon homogenizer at 1,000 r.p.m. PMSF $(200 \mu \mathrm{M})$ was added to prevent protein degradation. All subsequent steps were carried out at $0-4{ }^{\circ} \mathrm{C}$. The sample was centrifuged at $1,000 \mathrm{~g}$ for $15 \mathrm{~min}$ at $4{ }^{\circ} \mathrm{C}$, after which the pellet containing nuclei and cell debris (P1) was discarded. The supernatant (S1) was further centrifuged $\left(12,000 \mathrm{~g}, 15 \mathrm{~min}, 4^{\circ} \mathrm{C}\right)$ and then subjected to an additional cycle of resuspension and centrifugation for washing. The resulting pellet (P2, crude CG fraction) was resuspended in 300-mM sucrose buffer and loaded on top of a continuous sucrose gradient (from $300 \mathrm{mM}$ to $2.0 \mathrm{M}$ ) to remove other contaminants, including mitochondria. CGs were collected from the pellet after centrifugation at 27,000 r.p.m. for $60 \mathrm{~min}$ in a Beckman SW 41 Ti rotor and resuspended with the buffer (120 mM potassium glutamate, $20 \mathrm{mM}$ potassium acetate, $20 \mathrm{mM}$ HEPES and $\mathrm{KOH}(\mathrm{pH} 7.4)$ ). The fraction directly on top of the pellet was removed, and the pellet alone was resuspended only to purify mature CGs.

Synaptic vesicles from rat brain were purified as previously described ${ }^{18}$. Briefly, rat brains were homogenized in homogenization buffer supplemented with protease inhibitors, using a glass-Teflon homogenizer, with 10 strokes at 900 r.p.m. The homogenate was centrifuged for $10 \mathrm{~min}$ at $1,000 \mathrm{~g}$, and the resulting supernatant was further centrifuged for $15 \mathrm{~min}$ at $15,000 \mathrm{~g}$. The supernatant $\mathrm{S} 2$ was stored on ice for later use. The synaptosome pellet was lysed by addition of ice-cold water, and three strokes at 2,000 r.p.m. were applied. Protease inhibitors and HEPES were added to the lysate immediately. The lysate was centrifuged for $15 \mathrm{~min}$ at $17,000 \mathrm{~g}$, and the supernatant LS1 was combined with the S2. The mixture of LS1 and S2 was centrifuged for $25 \mathrm{~min}$ at $48,000 \mathrm{~g}$. The resulting supernatant CS1 was overlaid onto a $0.7-\mathrm{M}$ sucrose cushion and centrifuged for $1 \mathrm{~h}$ at $133,000 \mathrm{~g}$. The pellet was resuspended in column buffer (100 mM Tris$\mathrm{HCl}, 100 \mathrm{mM} \mathrm{KCl}$ (pH 7.4)) and loaded onto a Sephacryl S-1000 size-exclusion chromatography column $(100 \times 1 \mathrm{~cm})$.

Protein purification. All SNARE constructs were based on rat sequences and were cloned in the pET28a vector. The TeNT light chain (both wild-type and the inactive E234A mutant ${ }^{50}$ ), and SNARE proteins including the soluble form of synaptobrevin lacking the transmembrane domain $\left(\mathrm{Syb}_{1-96}\right)$ and $\mathrm{C} 2 \mathrm{AB}$ domain of synaptotagmin-1 (residues 97-421), were expressed in Escherichia coli and purified by $\mathrm{Ni}^{2+}{ }_{-} \mathrm{NTA}$ affinity chromatography followed by ion-exchange chromatography with a Mono S column on an Äkta system (GE Healthcare, Piscataway, $\mathrm{NJ})$. The stabilized Q-SNARE complex, referred to as the $\Delta \mathrm{N}$ complex and containing syntaxin-1A (183-288), SNAP-25A (with all cysteines replaced by alanines) and the C-terminal synaptobrevin fragment (49-96), was purified as described previously ${ }^{16}$. The 2:1 binary Q-SNARE complex containing syntaxin$1 \mathrm{~A}(1-288)$ and SNAP-25A (no cysteine) was expressed using cotransformation ${ }^{51}$. The $\Delta \mathrm{N}$ complex, the syntaxin-1A-SNAP-25A 2:1 binary complex, SNAP-25A (no cysteine) and syntaxin-1A (1-288, 183-288 and 183-262 (SyxH3)) were purified by $\mathrm{Ni}^{2+}$-NTA affinity chromatography followed by ion-exchange chromatography on a Mono Q column (GE Healthcare, Piscataway, NJ) in the presence of $50 \mathrm{mM}$ N-octyl- $\beta$-D-glucoside.

For anisotropy measurements, point-mutated C2AB (S342C) ${ }^{35}$ and $\mathrm{Syb}_{49-96}$ (T79C) in the $\Delta \mathrm{N}$ complex were labeled with Alexa Fluor 488 C5 maleimide.

Preparation of proteoliposomes. Unless indicated otherwise, the lipid composition of proteoliposomes (molar ratios) consists of $45 \%$ PC
(L- $\alpha$-phosphatidylcholine), 15\% PE (L- $\alpha$-phosphatidylethanolamine), 10\% PS (L- $\alpha$-phosphatidylserine), $25 \%$ cholesterol and 5\% PI ( $\mathrm{L}$ - $\alpha$-phosphatidylinositol). $\mathrm{PI}(4,5) \mathrm{P}_{2}$ or PI3P, at the indicated concentrations, replaced PI. For FRET-based dequenching assays, 1.5\% 1,2-dioleoyl-sn-glycero-3-phosphoethanolamine- $N$ (7-nitrobenz-2-oxa-1,3-diazol-4-yl) (NBD-DOPE) and 1.5\% 1,2-dioleoyl-snglycero-3-phosphoethanolamine- $N$-lissamine rhodamine B sulfonyl ammonium salt (Rhodamine-DOPE) were used as a donor and an acceptor dye, respectively. Synaptobrevin- and synaptotagmin-1-containing liposomes consist of 50\% PC, $20 \%$ PE, $20 \%$ PS and $10 \%$ cholesterol. When $15 \%$ PS or $10 \%$ PS was used, PC contents were adjusted accordingly.

As described ${ }^{52}$, liposomes were extruded using polycarbonate membranes of pore size $100 \mathrm{~nm}$ (Avanti Polar lipids) to give uniformly distributed large unilamellar vesicles (LUVs) in the diameter range of $100 \mathrm{~nm}$ as confirmed by fieldflow fractionation coupled with multiangle laser light scattering (FFF-MALLS, Wyatt Technology Corporation, Santa Barbara, CA, USA).

Incorporation of the proteins into liposomes was achieved by $n$-octyl- $\beta$-Dglucoside (OG)-mediated reconstitution. Proteoliposomes containing the stabilized acceptor complex ( $\triangle \mathrm{N}$ complex) or the syntaxin-1A-SNAP-25A binary complex in 2:1 stoichiometry (2:1 complex) were prepared by detergent-assisted insertion of proteins as described previously ${ }^{16,52} . \Delta \mathrm{N}$ complex in $50 \mathrm{mM}$ OG was mixed with LUVs (lipid-to-protein ratio of 500:1 (n/n)). In case of 2:1 complex incorporation with LUVs, lipid-to-protein ratio was 200:1. For content-mixing assays, $50 \mathrm{mM}$ calcein $(495 / 515 \mathrm{~nm}$ ) was encapsulated in proteoliposomes as described previously ${ }^{53}$. Lipids were dissolved in diethyl ether $(1.5 \mathrm{ml})$ and resuspended with $0.5 \mathrm{ml}$ of $50 \mathrm{mM}$ calcein $\left(2 \mathrm{Na}^{+}\right.$-calcein $\left.{ }^{2-}\right)$ in 20 - $\mathrm{mM}$ HEPES- $\mathrm{KOH}$ (pH 7.4), $75 \mathrm{mM} \mathrm{KCl}$ and $1 \mathrm{mM}$ DTT. Content mixing was specific to SNARE proteins incorporated in liposomes and leakage, determined by quenching calcein leaked into the medium by addition of $\mathrm{Co}^{2+}$, was only $4-5 \%$ of total calcein (for details see ref. 48).

Fusion reaction. CG fusion reactions were done at $37^{\circ} \mathrm{C}$. For each reaction, $50 \mu \mathrm{g}$ of CGs and $10 \mu \mathrm{l}$ of proteoliposomes were mixed in $1 \mathrm{ml}$ of buffer containing $120 \mathrm{mM}$ potassium glutamate, $20 \mathrm{mM}$ potassium acetate, $20 \mathrm{mM}$ HEPES-KOH ( $\mathrm{pH}$ 7.4) and $5 \mathrm{mM} \mathrm{MgCl}$. Unless indicated otherwise, acceptor liposomes contained the stabilized Q-SNARE complex, termed $\Delta \mathrm{N}$ complex $^{26}$. The 2:1 (syntaxin-1A-SNAP-25A) Q-SNARE complex was also tested for SNARE- and $\mathrm{Ca}^{2+}$-dependent fusion (Supplementary Fig. 4b). For $\mathrm{Ca}^{2+}$-dependent fusion, $5 \mathrm{mM} 2 \mathrm{Na}^{+}$-ATP was added. ATP should be made freshly for experiments because ATP is easily destroyed by freezing and thawing. Fluorescence dequenching signal was measured by FluoroLog and FluoroMax (HORIBA Jobin Yvon), with wavelengths of $460 \mathrm{~nm}$ (slit width of $1 \mathrm{~nm}$ ) for excitation and $538 \mathrm{~nm}$ (slit width of $3 \mathrm{~nm}$ ) for emission. Fluorescence values were normalized as the percentage value of the maximum donor fluorescence induced by $0.1 \%$ Triton X-100 detergent treatment at the end of each experiment. 'No addition' represents basal fusion without any treatment or $\mathrm{Ca}^{2+}$. Quantification of vesicle-fusion data of lipid-mixing and content-mixing assay is presented as a percentage by normalizing basal fusion after 20 min of reaction time. $\mathrm{Ca}^{2+}$-dependent fusion with the different concentrations of $\mathrm{PI}(4,5) \mathrm{P}_{2}$ (Fig. 3d) was normalized according to Fusion $\mathrm{nor}_{\text {no }}=$ $\left(T-T_{0}\right) /\left(T_{\max } T_{0}\right)$, where $T$ is the percentage of total fluorescence induced by vesicle fusion, $T_{0}$ indicates basal fusion without $\mathrm{Ca}^{2+}$ and $T_{\max }$ indicates fusion at $100 \mu \mathrm{M} \mathrm{Ca}^{2+}$ (maximum level).

Cryo-electron microscopy. Samples were bound in a Vitrobot Mark IV (FEI Company) to a glow-discharged carbon foil-covered grid. The suspension was blotted $2 \times$ for $1 \mathrm{~s}$ at blot force $=2$ and vitrified at $24{ }^{\circ} \mathrm{C}$ and $97 \%$ humidity. The samples were evaluated with a CM 120 transmission electron microscope, and pictures were taken with a TemCam 224A slow scan CCD camera (TVIPS, Gauting, Germany).

Fluorescence-anisotropy measurements. Anisotropy measurements were carried out in a FluoroLog 3 spectrometer in T-configuration equipped for polarization (Model FL322, Jobin Yvon). All experiments were done at $37^{\circ} \mathrm{C}$ in $1 \mathrm{ml}$ of buffer containing $120 \mathrm{mM}$ potassium glutamate, $20 \mathrm{mM}$ potassium acetate, $20 \mathrm{mM}$ HEPES-KOH (pH 7.4) and $5 \mathrm{mM} \mathrm{MgCl}_{2} \cdot 2 \mathrm{Na}^{+}$-ATP and $\mathrm{CaCl}_{2}$ were treated as indicated. Alexa Fluor 488-labeled proteins were excited at $488 \mathrm{~nm}$ (slit width of $8 \mathrm{~nm}$ ), and their emission was measured at $520 \mathrm{~nm}$ (slit width of $10 \mathrm{~nm}$ ). For monitoring of SNARE assembly, $200 \mu \mathrm{g}$ CGs were incubated with 
$1 \% \mathrm{PI}(4,5) \mathrm{P}_{2}$-containing liposomes that incorporate the $\Delta \mathrm{N}$ complex $\left(\mathrm{Syb}_{49-96}\right.$ labeled with Alexa Fluor 488 at T79C). For monitoring of the binding of the $\mathrm{C} 2 \mathrm{AB}$ domain, $30 \mathrm{nM} \mathrm{C} 2 \mathrm{AB}$ (S342C, Alexa Fluor 488-labeled) was incubated with $30 \mu \mathrm{g}$ CGs or protein-free liposomes containing $20 \%, 15 \%$ or $10 \%$ PS. The $G$ factor was calculated according to $G=I_{\mathrm{HV}} / I_{\mathrm{HH}}$, where $I$ is the fluorescence intensity, the first subscript letter indicates the direction of the exciting light, and the second subscript letter the direction of emitted light. The intensity of the vertically $(\mathrm{V})$ and horizontally $(\mathrm{H})$ polarized emission light after excitation by vertically polarized light was measured. The anisotropy $(r)$ was determined according to $r=\left(I_{\mathrm{VV}}-G \times I_{\mathrm{VH}}\right) /\left(I_{\mathrm{VV}}+2 G \times I_{\mathrm{VH}}\right)$.

$\mathrm{Ca}^{2+}$ calibration. ATP contains negatively charged oxygen atoms that bind to $\mathrm{Mg}^{2+}, \mathrm{Ca}^{2+}$ or $\mathrm{Sr}^{2+}$, thereby chelating divalent cations ${ }^{42} . \mathrm{Ca}^{2+}$ concentrations were calibrated with Fluo-5N, a low-affinity $\mathrm{Ca}^{2+}$ indicator with a $K_{\mathrm{d}}=90 \mu \mathrm{M}$, and experiment data were correlated with a simulation that calculates the free $\mathrm{Ca}^{2+}$ concentrations (http://maxchelator.stanford.edu).
Statistical analysis. All quantitative data are mean \pm s.d. from three or more independent experiments. Dose-response curves were fit using four-parameter logistic equations (4PL) to calculate $\mathrm{EC}_{50}$ (SigmaPlot).

49. Smith, A.D. \& Winkler, H. A simple method for the isolation of adrenal chromaffin granules on a large scale. Biochem. J. 103, 480-482 (1967).

50. Li, Y. et al. A single mutation in the recombinant light chain of tetanus toxin abolishes its proteolytic activity and removes the toxicity seen after reconstitution with native heavy chain. Biochemistry 33, 7014-7020 (1994).

51. Weber, T. et al. SNAREpins: minimal machinery for membrane fusion. Cell 92 , 759-772 (1998).

52. Cypionka, A. et al. Discrimination between docking and fusion of liposomes reconstituted with neuronal SNARE proteins using FCS. Proc. Natl. Acad. Sci. USA 106, 18575-18580 (2009).

53. Kendall, D.A. \& MacDonald, R.C. Characterization of a fluorescence assay to monitor changes in the aqueous volume of lipid vesicles. Anal. Biochem. 134, 26-33 (1983). 